\title{
How to prevent perioperative myocardial injury: The conundrum continues
}

Jian-Zhong Sun

Thomas Jefferson University

David Maguire

Thomas Jefferson University

Follow this and additional works at: https://jdc.jefferson.edu/anfp

Part of the Cardiology Commons

Let us know how access to this document benefits you

\section{Recommended Citation}

Sun, Jian-Zhong and Maguire, David, "How to prevent perioperative myocardial injury: The conundrum continues" (2007). Department of Anesthesiology Faculty Papers. Paper 1.

https://jdc.jefferson.edu/anfp/1

This Article is brought to you for free and open access by the Jefferson Digital Commons. The Jefferson Digital Commons is a service of Thomas Jefferson University's Center for Teaching and Learning (CTL). The Commons is a showcase for Jefferson books and journals, peer-reviewed scholarly publications, unique historical collections from the University archives, and teaching tools. The Jefferson Digital Commons allows researchers and interested readers anywhere in the world to learn about and keep up to date with Jefferson scholarship. This article has been accepted for inclusion in Department of Anesthesiology Faculty Papers by an authorized administrator of the Jefferson Digital Commons. For more information, please contact: JeffersonDigitalCommons@jefferson.edu. 


\title{
How to prevent perioperative myocardial injury: The conundrum continues
}

\author{
Jian-Zhong Sun, MD, PhD, and David Maguire, MD \\ Department of Anesthesiology, Suite G8490 \\ Jefferson Medical College \\ Thomas Jefferson University \\ 111 S. $11^{\text {th }}$ Street \\ Philadelphia, PA \\ Email: Jian-Zhong Sun@jefferson.edu
}

This work was supported in part by a Foundation for Anesthesia Education and Research grant (J.Z.S.).

\section{Abstract \\ Background}

Perioperative myocardial injury (PMI) remains a major cause of perioperative morbidity and mortality but clinical strategies to prevent PMI are still uncertain.

\section{Methods and Results}

We comprehensively searched PubMed for major research articles concerning clinical strategies to prevent PMI. The key findings are as follows: (1) the American College of Cardiology/American Heart Association guideline update for perioperative cardiovascular evaluation for noncardiac surgery is very useful to stratify cardiac risk preoperatively; (2) cardiac troponin has emerged as a biomarker to diagnose postoperative PMI and to predict clinical outcomes; (3) coronary revascularization before noncardiac surgery probably would provide cardiac protection in select patients, especially in patients with high-risk coronary artery disease; (4) elective noncardiac surgery should be postponed in patients who received coronary stenting recently because of high incidence of serious cardiac complications (minimum 68 weeks for bare metal stents and 6-12 months for drug-eluting stents); and (5) $\beta$-blockers and statins are very promising drugs and probably would prevent PMI in a select patient population, especially in patients with intermediate risk and stable coronary artery disease.

\section{Conclusions}

Further studies, especially randomized clinical trials and mechanistic investigation are

This paper has been peer reviewed. It is the authors' final version prior to publication in The American Heart Journal 154(6):1031-1028, December 2007. The published version is available at http://dx.doi.org/10.1016/j.ahj.2007.07.025, copyright by Mosby, Inc. 
needed to find the best and effective clinical strategies to prevent/reduce PMI. (Am Heart J 2007;154:1021-8.)

Perioperative myocardial injury (PMI), including myocardial ischemia, cardiac dysfunction, cardiac arrhythmias, myocardial infarction, and cardiac arrest continues to be a major challenge to perioperative physicians because its incidence has not substantially decreased for the past 2 decades. ${ }^{1,2}$ Despite extensive clinical and basic research, the mechanisms responsible for PMI remain enigmatic. Currently, the predominant theories are that PMI may be caused by prolonged stress- induced myocardial ischemia, atherosclerotic plaque rupture, or a combination of the two. Clinically, perioperative myocardial ischemia and infarction may present differently; pathologically, they are all secondary to alterations of coronary plaque morphology and function and/or the loss of balance between myocardial oxygen supply and demand, in which inflammation has been linked with the development of atherosclerotic disease and instability and may cause acute coronary syndromes (ACSs) and PMI.

The potential triggers for PMI include surgical stress, catecholamine release, and inflammatory reaction; prolonged/repeated stress or extreme surgical stress especially may inflict inflammation and induce repeated/prolonged myocardial ischemia, which may serve as a primary cause for PMI, including perioperative myocardial infarction. Several lines of evidence support this hypothesis. First, perioperative cardiac complications are preceded almost universally by long-rather than short- duration ST-segment changes-an indication of pro-longed myocardial ischemia. ${ }^{3,4}$ Second, most perioperative myocardial infarctions occur early after surgery (most stressful time) and are asymptomatic ("silent" myocardial infarction) 5,6 ; most of them are preceded by episodes of increases in heart rate $^{4,6}$ and show non-Q wave rather than $\mathrm{Q}$ wave myocardial infarction, 3,5 whereas clinical studies have well demonstrated that complete coronary occlusion is infrequently observed in patients with non-Q wave myocardial infarction (only 26\%-42\%) compared with patients with Q wave infarction $(\geq 84 \%){ }^{7}$ Third, $\beta$-adrenergic receptor blockers, because of their inherent role of blocking sympathetic activation, have been shown to reduce perioperative ischemia and the risk of myocardial infarction and death in high-risk surgical patients (see below). Finally, our recent study demonstrated that chronic catecholamine stimulation can aggravate myocardial injury by provoking inflammatory reaction and increasing myocardial apoptosis, thereby illustrating a mechanistic link between extreme/prolonged surgical stress $\rightarrow$ catecholamine release $\rightarrow$

inflammation/myocardial apoptosis and PMI. ${ }^{8}$

Nevertheless, coronary plaque disruption was found in more than half of fatal perioperative myocardial infarctions and was considered as a primary cause of fatal 
perioperative myocardial infarction. ${ }^{9,10}$ More likely, surgery, especially major surgery with its associated trauma, anesthesia, pain, bleeding, and hypothermia, is comparable to an extreme stress test, thereby producing a triggering/provoking effect in the pathogenesis of PMI (ie, inflicting inflammation and destabilizing plaques) and ultimately leading to appearance of PMI.

Clinical strategies to prevent PMI have been evolving greatly. In 1977, Goldman et al pioneered the concept of a risk index to account for the multifactorial nature of contributors to risk of cardiac morbidity, which has led to the landmark development in perioperative medicine, that is, the American College of Cardiology (ACC)/ American Heart Association (AHA) guidelines for perioperative cardiovascular evaluation for noncardiac surgery in $1996^{12}$ and an update in 2002 . $^{13}$ These guidelines focused on preoperative testing to identify patients with significant coronary artery disease (CAD) and subsequent coronary revascularization in select patients. The guidelines currently play a major role in perioperative medicine. However, because of the poor positive predictive value of noninvasive cardiac stress tests, the controversy about the benefit of coronary revascularization before noncardiac surgery, and the considerable risk of coronary angiography and coronary revascularization in high-risk patients, perioperative physicians have been continuously searching for alternative approaches to prevent/reduce perioperative cardiac complications. In 1996, Mangano et al performed a randomized clinical trial (RCT) to investigate the effect of the $\beta$-blocker, atenolol, on patient outcomes and concluded that in patients with risk for CAD who must undergo noncardiac surgery, treatment with atenolol during hospitalization can reduce mortality and the incidence of cardiovascular complications for as long as 2 years after surgery. In 2003, Poldermans et al ${ }^{15}$ provided evidence in a case-controlled study that statin use reduces perioperative mortality in patients undergoing major vascular surgery. More recently, the ACC/ AHA updated their recommendations on perioperative $\beta$ blocker therapy. ${ }^{16}$ These significant developments in perioperative medical therapy to prevent/reduce PMI have shifted the interest in perioperative cardiac care greatly, from risk stratification and potential coronary revascularization to risk modification with $\beta$-blockers and/or statins. Nevertheless, the debate and controversy exist in almost every aspect of clinical strategies to prevent PMI. These strategies include mainly preoperative cardiac risk assessment, perioperative monitoring, prophylactic coronary revascularization, coronary stents, and perioperative medical therapy.

\section{Preoperative cardiac risk assessment}

The ACC/AHA guideline update for perioperative cardiovascular evaluation for noncardiac surgery can help to stratify cardiac risk and identify the patients who need preoperative interventions to reduce cardiac risk. ${ }^{13}$ The rationale for intensive screening is based on the assumption that coronary revascularization prevents PMI in patients with $\mathrm{CAD}$. As the ACC/AHA task force stated in the guideline: "A large proportion of the data 
used to develop these guidelines are based on observational or retrospective studies or knowledge of management of cardiovascular disorders in the nonoperative setting. Although the collective body of knowledge about the identification of high-and low-risk patients by perioperative clinical and noninvasive evaluation is substantial, the number of prospective or randomized studies that have been performed to establish the value of different treatments on perioperative outcomes is small." ${ }^{13}$ Based on the guidelines, many patients undergo preoperative testing for detection of CAD before noncardiac surgery. However, noninvasive cardiac stress testing for perioperative myocardial infarction or death has very poor positive predictive values (b20\%), although it has excellent negative predictive values (near $100 \%$ ). Therefore, there is uncertainty and much debate on how to prepare surgery for the patient who has positive results from preoperative cardiac stress testing, that is, medical therapy versus coronary revascularization. In addition, very few RCTs support either of the treatments (see below).

The Lee et al ${ }^{18}$ revised cardiac risk index is a simple and practical clinical risk index. It includes 6 variables to identify patients at high risk for perioperative cardiac complications: history of CAD, history of congestive heart failure, history of cerebral vascular accident, preoperative insulin treatment, serum creatinine level N2.0 mg/dL, and high-risk surgical procedure. The presence of 3 or more of these risk variables (class III and IV) conferred an event rate as high as $11 \%$ in a group of 1422 patients, whereas the event rate was b1\% in the presence of one or none of these variables (class I and II).

Other investigators also approached the problem of assessing PMI risk through the development of multivariate models; they proposed that the expected risk from these risk indices may vary along institutions ${ }^{19}$ and a test required by these indices should only be performed if this has consequences on perioperative management. And this is why routine electrocardiogram and chest $\mathrm{x}$-ray have been abandoned as preoperative tests in low- and intermediate-risk surgical patients without specific indications.

\section{Perioperative monitoring}

Cardiac troponin I (cTnI) and cTnT have become the biomarkers of choice to diagnose myocardial infarction and to risk-stratify patients with suspected ACS. A recent study by Wong et al ${ }^{20}$ demonstrated that cTn elevations were associated with a higher risk of multi-vessel disease, complex lesion morphology, and visible thrombus on coronary angiography. Moreover, such patients had more impairment in microvascular function, thereby suggesting a greater propensity for distal embolization of plaque material to the microvasculature. Recently, intravascular ultrasound studies have illustrated that those patients with ACS and elevated troponin levels had greater atheroma burden at the lesion site, more reference segment atherosclerosis, and more frequent findings compatible with 
thrombus at the lesion compared with patients who did not have troponin elevation. Taken together, these findings likely explain the consistent association between troponin elevation, even at low levels, and recurrent ischemic events in patients with ACS.

In surgical patients, Landesberg et al ${ }^{22}$ and Kim et al ${ }^{23}$ have demonstrated that postoperative cTn measurements can detect postoperative myocardial infarction and predict short-term (6 months) and long-term (1-5 years) survival after vascular surgery. Recently, in a study with intense postoperative cTnI surveillance in 1136 patients who underwent abdominal aortic surgery, Le Manach et al revealed 2 types of PMI according to the time of appearance and rate of increase in cTnI: acute (b24-hour) and early increases of cTnI above threshold may indicate acute coronary occlusion for early morbidity; prolonged low-level cTnI release followed by a delayed (N24 hours) increase of cTnI may lead to prolonged myocardial ischemia for later events. They proposed monitoring perioperative cTnI concentrations and early institution of treatment for patients with increased cTnI before it leads to irreversible necrosis.

Recently, Mahla et al ${ }^{25}$ investigated the use of cardiac stress marker, N-terminal pro-brain natriuretic peptide (NT-proBNP), to stratify PMI risk in patients undergoing vascular surgery and found that a single postoperative NT-proBNP determination provides important additional prognostic information for inhospital and late cardiac events and therefore may support therapeutic decisions to prevent subsequent myocardial damage. This study again provides evidence that the extent of surgical stress is closely related with risk of PMI.

Despite the progress in clinical application of biomarkers for myocardial injury, such as cTn as a marker of myocardial necrosis, there is still a lack of markers of myocardial ischemia, especially the biomarkers that

(1) can detect myocardial ischemia without myocardial necrosis,

(2) increase the size of release proportional to the extent of ischemia,

(3) appear in circulation rapidly after onset of ischemia and last long enough for the detection, and

(4) are easy to measure with high sensitivity and reasonable specificity.

\section{Prophylactic coronary revascularization}

The role of prophylactic/preoperative coronary revascularization in patients with CAD before noncardiac surgery has been examined carefully in several clinical studies but remains controversial. ACC/AHA guidelines update recommended coronary revascularization only for subgroups of high-risk patients with unstable cardiac symptoms or those for whom coronary artery revascularization offers a long-term benefit, independent of the need for noncardiac surgery. This recommendation was based predominately on the $\mathrm{CASS}^{26}$ as follows. 


\section{Coronary artery bypass graft before noncardiac surgery}

In 1997, the CASS investigators reported their study, which is the largest study to date and included 3368 noncardiac operations performed within a 10-year period among patients assigned to medical therapy or coronary artery bypass graft (CABG). In this study they found that among 1961 patients undergoing higher risk surgery (involving the thorax, abdomen, vasculature, and head and neck), prior CABG was associated with fewer postoperative deaths $(1.7 \%$ vs $3.3 \%, \mathrm{P}=.03)$ and myocardial infarctions $(0.8 \%$ vs $2.7 \%, \mathrm{P}$ $=.02$ ) compared with medically managed CAD. There was no difference in the outcome of patients undergoing low-risk procedures such as breast and urologic surgery. They concluded that "in patients with known CAD, noncardiac surgeries involving the thorax, abdomen, vasculature, and head and neck are associated with the highest cardiac risk, which is reduced among patients with prior CABG." ${ }^{26}$ However, major limitations of the CASS are that the mortality and morbidity associated with CABG was not factored into the analysis of perioperative outcomes and that perioperative medical treatments, such as perioperative $\beta$-blocker and statin therapy, have undergone major improvements since the study was published 10 years ago.

\section{Coronary revascularization before vascular surgery}

In the CARP trial, however, McFalls et al ${ }^{27}$ reported that patients with stable CAD who were scheduled for elective vascular operations at 18 Veterans Affairs hospitals were randomly assigned to undergo coronary revascularization (CABG or percutaneous coronary intervention [PCI]) or medical therapy. After the vascular surgery, there were no differences between the 2 groups in the incidence of myocardial infarction or mortality (a median follow-up of 2.7 years), and therefore coronary revascularization by either CABG or PCI before elective vascular surgery cannot be recommended in patients with stable CAD. This randomized study provided evidence that coronary revascularization in patients with stable CAD does not provide better protection when compared to current medical therapy, which included $\beta$-blockers and statins. The study, however, excluded patients with symptoms of unstable coronary disease, left main CAD, aortic stenosis, or severe left ventricular dysfunction, which are class I indications for CABG based on ACC/AHA guidelines for CABG surgery published in 1999.

In contrast, other studies by Eagle et al, ${ }^{26}$ Landersberg et al, ${ }^{29}$ and Garofalo et al have shown that previous coronary revascularization provides protection against adverse cardiac events and mortality after vascular surgery. The causes responsible for the difference between the above studies are unclear but probably are due to patient populations included in those studies being different. Taken together, for upcoming surgery, patients with high-risk CAD (left main or 3-vessel disease, poor left ventricular function, and/or diabetes) probably will benefit from coronary revascularization, whereas 
patients with intermediate-risk and stable CAD will be more likely to do better with optimal medical therapy.

\section{CABG versus PCI (angioplasty or stenting) before vascular surgery}

In the substudy of the CARP trial, Ward et al ${ }^{31}$ compared clinical outcomes in patients receiving $\mathrm{CABG}$ versus $\mathrm{PCI}$ as prophylaxis for elective vascular surgery and found that compared with patients with PCI (131 patients), patients with CABG (91 patients) had fewer myocardial infarctions despite more diseased vessels in the CABG group and tended to spend less time in the hospital after the vascular operation. The authors concluded that more complete revascularization accounted for the intergroup differences.

\section{CABG versus coronary angioplasty before noncardiac surgery}

Previous studies have shown that $\mathrm{CABG}$ reduces the risk of cardiac complications after noncardiac surgery. Whether coronary angioplasty provides equivalent protection is not known. In BARI, Hassan et al ${ }^{32}$ found that rates of myocardial infarction and death after noncardiac surgery are similarly low after contemporary bypass surgery or coronary angioplasty in patients with multi-vessel CAD in a randomized study.

\section{Coronary stents in surgical patients}

Percutaneous transluminal coronary angioplasty (PTCA) was introduced by Gruntzing in 1977. Sigwart and Puel deployed the first coronary stent in humans in 1986. By 1999, stenting or bare metal stents (BMSs) composed $84.2 \%$ of all PCIs. In 2001, the drugeluting stent (DES) was introduced and 2 years later it was approved by the Food and Drug Administration in the United States. At present, most coronary stents have been converted to DES in the United States. PTCA, coronary artery stent, and DES are 3 landmark developments in interventional cardiology. However, despite the initial enthusiasm that resulted in the advent of DES, incomplete endothelialization and stent thrombosis continue to plague these devices. Initial studies on animals demonstrated complete endothelialization with BMS at 28 days, whereas DES uniformly showed incomplete healing at 180 days.

In 2000, Kaluza et al ${ }^{36}$ first reported on 40 patients treated with BMS who underwent noncardiac surgery within 6 weeks of stent implantation. Seven patients had myocardial infarction, of which 6 were fatal. Other similar studies have also shown a high incidence of cardiovascular complications when noncardiac surgery was performed shortly after coronary stenting or PTCA alone. 
The 2007 ACC/AHA for Cardiovascular Angiography and Interventions recommendations for the prevention of stent thrombosis after coronary stent implantation state that, at a minimum, patients should be treated with clopidogrel $75 \mathrm{mg}$ and aspirin 325 mg for 1 month after BMS implantation, 3 months after sirolimus DES implantation, 6 months after paclitaxel DES implantation, and ideally, up to 12 months if they are not at high risk for bleeding. This advisory stresses the importance of 12 months of dual antiplatelet therapy after placement of a DES and educating the patient and health care providers about hazards of premature discontinuation. It also recommends postponing elective surgery for 1 year, and if surgery cannot be deferred, considering the continuation of aspirin during the perioperative period in high-risk patients with DES. Nevertheless, in a recent prospective observational study from 3 medical institutions, Vicenzi et al reported that despite all patients receiving continuous heparin and antiplatelet drugs or only shortly discontinued, nearly half of the patients (46 of $103,44.7 \%$ ) who received coronary artery stents within 1 year had complications after surgery; $4.9 \%$ of the patients died. All but 2 adverse events (bleeding only) were of a cardiac nature.

At present, for the patients with PCI who require surgery, there is still a lack of consensus regarding how much time should pass between PCI and noncardiac surgery. However, based on the ACC/ AHA recommendations as above, elective noncardiac surgery should be postponed in patients who recently received coronary stenting for a probable minimum of 6 to 8 weeks for BMS and 6 to 12 months for DES. More recently, based on a clinical study in 60 patients, Ingraldi et al proposed that although DES is effective in keeping open diseased heart arteries, they should not be used for patients who need to have noncardiac surgery a short time after an interventional heart procedure; instead, BMS provides a safer choice for these patients (abstract at the 2007 Scientific Session of the Society for Cardiovascular Angiography and Interventions).

\section{Perioperative medical therapy}

In recent years, significant progress has been made in medical treatments of cardiovascular diseases. Along with the progress, medical interventions with drugs such as $\beta$-blockers and statins have been shown to reduce the occurrence of perioperative cardiac complications, which has brought in the surge of interest on risk modification with medical therapies instead of risk stratification with potential coronary revascularization in surgical patients.

\section{$\beta$-Blocker therapy}

The ACC/AHA 2006 Guideline Update on Perioperative $\beta$-Blocker Therapy ${ }^{16}$ is a formal 
position statement indicating that performance measures should be limited to class I or class III recommendations - those recommendations in which patients should or should not have the form of therapy-and that they should not include class IIa or IIb recommendations, in which the evidence is less strong and for which opinion dictates the class of indications. Class I recommendation includes the following: (1) $\beta$-blockers should be continued in patients undergoing surgery who are receiving $\beta$-blockers to treat angina, symptomatic arrhythmias, hypertension, or other ACC/AHA class I guideline indications; (2) $\beta$-blockers should be given to patients undergoing vascular surgery at high cardiac risk owing to the finding of ischemia on preoperative testing. Class III recommendation states that $\beta$-blockers should not be given to patients undergoing surgery who have absolute contra-indications to $\beta$-blockade.

The best protocol for administration of perioperative $\beta$-blockers remains uncertain, although previous studies have demonstrated that tight heart rate control by $\beta$-blockers reduced perioperative myocardial ischemia and improved clinical outcomes.

\section{Statin therapy}

Statin therapy is well established for prevention of cardiovascular disease. Statins may also reduce postoperative mortality and morbidity via a pleiotropic (non- lipid-lowering) effect. Recently, Hindler et al ${ }^{43}$ conducted a meta-analysis to evaluate the overall effect of preoperative statin therapy on postoperative outcomes. They analyzed the data from 12 retrospective and 3 prospective trials with a total of 223010 patients and found that preoperative statin therapy was associated with $38 \%$ and $59 \%$ reduction in the risk of mortality after cardiac and vascular surgery, respectively. When including noncardiac surgery, a $44 \%$ reduction in mortality was observed.

Nevertheless, so far there is only one RCT on the effect of statin in patients undergoing noncardiac surgery carried out in Brazil. In this study with 50 patients in the treatment and 50 in the placebo group, respectively, short-term (45 days) treatment with atorvastatin significantly reduced the incidence of major adverse cardiovascular events after vascular surgery; that is, only 4 cases in the atorvastatin group had adverse events compared with 17 cases in the placebo group $(8.0 \%$ vs $26.0 \%, \mathrm{P}=.031)$. Despite the very positive findings from this study, the trial was small and the treatment effect appears very large (69\% relative risk reduction), which is inconsistent with the results from other RCTs of statins in preventing cardiovascular events.

\section{$\alpha-2-A d r e n e r g i c$ agonist therapy}

$\alpha$-2-Adrenergic agonists, such as clonidine, can be used in those intolerant to $\beta$-blocker therapy and have been shown to significantly reduce perioperative myocardial ischemia 
and postoperative death.

\section{Aspirin therapy}

Aspirin, as an anti-inflammatory and antiplatelet medicine, has been widely used for longterm prevention of cardiovascular disease. However, in perioperative medicine, there are still no data to support use of aspirin to prevent PMI. Periprocedural withdrawal of aspirin may increase cardiovascular complications including ACS, but continuing aspirin increases perioperative bleeding by about 1.5 fold. ${ }^{46}$ Preoperative and early postoperative aspirin therapy has been reported to improve postoperative outcomes in patients undergoing $\mathrm{CABG} .{ }^{48}$ In patients with vascular surgery, Robless et al conducted a metaanalysis of clinical trials of antiplatelet therapy and found that aspirin reduced the incidence of myocardial infarction, stroke, and vascular death but the benefit did not reach statistical significance. In contrast, in a large, multinational RCT on aspirin therapy as prophylaxis for patients undergoing hip fracture surgery, ischemic cardiac events (myocardial infarction or death) were found to be higher among patients who received aspirin than placebo (hazard ratio $1.33, \mathrm{P}=.05)^{50}$

Despite the progress that has been made, several recent clinical trials (MaVS study, DIPOM trial, ${ }^{52}$ and POBBLE trial ${ }^{53}$ ) have failed to demonstrate the use of $\beta$-blockers in the reduction of perioperative cardiac complications, and raised questions: Where did we go wrong and shall we reverse the ACC/AHA recommendations on perioperative $\beta$ blockers? ${ }^{54}$ Further clinical trials (ie, POISE trial, and DECEASE-IV study ${ }^{56}$ ) are ongoing, which may or may not clarify these controversies because the target population, the choice of $\beta$-blockers, surgical procedures, and other options of the therapy may be different. In addition, it should be appreciated that even superbly designed RCTs may be inapplicable to large numbers of patients because the patients enrolled may not represent the patient population or the patients excluded would normally receive the treatment of interest. Nevertheless, those trials will certainly have important implications on whether or how to give $\beta$ blockers to perioperative patients.

In the broad field of clinical medicine, previous clinical trials have clearly demonstrated that $\beta$-blockers provide multiple benefits to patients with CAD and heart failure, such as decreasing mortality in patients with acute myocardial infarction, secondary prevention of myocardial infarction in post-myocardial infarction patients, significant reduction in arrhythmic death or cardiac death in post-myocardial infarction patients, and improving survival in patients with heart failure. In a recent multinational cohort study that included 44372 patients with ACS in 14 countries, Fox et al ${ }^{58}$ demonstrated that improvements in the management of patients with ACS, including increases in use of $\beta$-blockers, are accountable for a significant decline in rates of new heart failure and mortality and in rates 
of stroke and myocardial infarction at 6 months. In the field of surgery and anesthesiology, Lindenauer et al conducted a very large retrospective cohort study including 663635 patients from 329 hospitals throughout the United States. In this study, they found that perioperative administration of $\beta$-blockers was associated with a reduced risk of death in the hospital among high-risk, but not low-risk, patients undergoing major noncardiac surgery, indicating a potential interaction between the effect of $\beta$-blockers and the extent of CAD risk.

Because of the overwhelming number of studies, some with conflicting results, we probably should consider the evidence in 2 dimensions, internal validity (RCTs) and external validity (retrospective and/or observational studies), and recognize that different methods may complement one another. Thus, the intervention adapted ideally should be supported by evidence with both high internal and high external validity.

In summary, either $\beta$-blockers or prophylactic coronary revascularization more likely would provide cardiac protection in select patient populations, that is, $\beta$-blockers for patients with intermediate-risk and stable CAD, and prophylactic coronary revascularization for patients with high risk of CAD. On the other hand, statins have proven their therapeutic value for a wide range of patients with CAD; up to now, statins have also appeared to provide cardiac protection for surgical patients. Nevertheless, given that the present RCTs with statins in surgical patients is limited to one small trial in which $\leq 100$ patients have actually been treated ${ }^{43,44}$ and given that ACC/AHA/National Heart, Lung, and Blood Institute clinical advisory on the use and safety of statins has concluded that it may be prudent to withhold statins during hospitalization for major surgery because of potential side effects such as myopathy, it is still premature to recommend the routine use of statins for all surgical patients with cardiac risk.

Years have passed since the cardiac risk index was proposed in 1977; however, the PMI conundrum continues. The pathogenetic mechanisms responsible for PMI remain a mystery, which hinders us greatly from developing effective clinical strategies to prevent/ reduce PMI.

We thank Drs. Grunwald and Seltzer for their critical review of the manuscript.

\section{References}

1. Devereaux PJ, Goldman L, Cook DJ, et al. Perioperative cardiac events in patients undergoing noncardiac surgery: a review of the magnitude of the problem, the pathophysiology of the events and methods to estimate and communicate risk. 
CMAJ 2005;173: 627-34.

2. Mangano DT. Perioperative cardiac morbidity. Anesthesiology 1990;72:153-84.

3. Landesberg G, Luria MH, Cotev S, et al. Importance of long-duration postoperative ST-segment depression in cardiac morbidity after vascular surgery. Lancet 1993;341:715-9.

4. Landesberg G, Mosseri M, Zahger D, et al. Myocardial infarction after vascular surgery: the role of prolonged stress-induced ST depression-type ischemia. J Am Coll Cardiol 2001;37:1839-45.

5. Badner NH, Knill RL, Brown JE, et al. Myocardial infarction after noncardiac surgery. Anesthesiology 1998;88:572-8.

6. McCann RL, Clements FM. Silent myocardial ischemia in patients undergoing peripheral vascular surgery: incidence and association with perioperative cardiac morbidity and mortality. J Vasc Surg 1989;9:583-7.

7. DeWood MA, Stifter WF, Simpson CS, et al. Coronary arteriographic findings soon after non-Q-wave myocardial infarction. N Engl J Med 1986;315:417-23.

8. Hu A, Jiao X, Gao E, et al. Chronic beta-adrenergic receptor stimulation induces cardiac apoptosis and aggravates myocardial ischemia/reperfusion injury by provoking inducible nitric-oxide synthase-mediated nitrative stress. J Pharmacol Exp Ther 2006;318: 469-75.

9. Dawood MM, Gutpa DK, Southern J, et al. Pathology of fatal perioperative myocardial infarction: implications regarding pathophysiology and prevention. Int J Cardiol 1996;57:37-44.

10. Buffington CW, Perez WJ. Perioperative myocardial ischemia and infarction. Atherosclerotic plaque rupture in coronary artery disease. Int Anesthesiol Clin 1992;30:217-35.

11. Goldman L, Caldera DL, Nussbaum SR, et al. Multifactorial index of cardiac risk in noncardiac surgical procedures. N Engl J Med 1977;297:845-50.

12. Eagle KA, Brundage BH, Chaitman BR, et al. Guidelines for perioperative cardiovascular evaluation for noncardiac surgery. Report of the American College of Cardiology/American Heart Association Task Force on Practice Guidelines. Committee on Perioperative Cardiovascular Evaluation for Noncardiac Surgery. Circulation 1996;93:1278-317.

13. Eagle KA, Berger PB, Calkins H, et al. ACC/AHA guideline update for perioperative cardiovascular evaluation for noncardiac surgery- executive 
summary: a report of the American College of Cardiology/ American Heart Association Task Force on Practice Guidelines (Committee to Update the 1996 Guidelines on Perioperative Cardiovascular Evaluation for Noncardiac Surgery). Circulation 2002;105:1257-67.

14. Mangano DT, Layug EL, Wallace A, et al. Effect of atenolol on mortality and cardiovascular morbidity after noncardiac surgery. Multicenter study of perioperative ischemia research group. N Engl J Med 1996;335:1713-20.

15. Poldermans D, Bax JJ, Kertai MD, et al. Statins are associated with a reduced incidence of perioperative mortality in patients undergoing major noncardiac vascular surgery. Circulation 2003;107:1848-51.

16. Fleisher LA, Beckman JA, Brown KA, et al. ACC/AHA 2006 guideline update on perioperative cardiovascular evaluation for noncardiac surgery: focused update on perioperative beta-blocker therapy: a report of the American College of Cardiology/American Heart Association Task Force on Practice Guidelines (Writing Committee to Update the 2002 Guidelines on Perioperative Cardiovascular Evaluation for Noncardiac Surgery): developed in collaboration with the American Society of Echocardiography, American Society of Nuclear Cardiology, Heart Rhythm Society, Society of Cardiovascular Anesthesiologists, Society for Cardiovascular Angiography and Interventions, and Society for Vascular Medicine and Biology. Circulation 2006;113:2662-74.

17. Eagle KA, Lau WC. Any need for preoperative cardiac testing in intermediate-risk patients with tight beta-adrenergic blockade? J Am Coll Cardiol 2006;48:970-2.

18. Lee TH, Marcantonio ER, Mangione CM, et al. Derivation and prospective validation of a simple index for prediction of cardiac risk of major noncardiac surgery. Circulation 1999;100:1043-9.

19. Detsky AS, Abrams HB, McLaughlin JR, et al. Predicting cardiac complications in patients undergoing non-cardiac surgery. J Gen Intern Med 1986;1:211-9.

20. Wong GC, Morrow DA, Murphy S, et al. Elevations in troponin T and I are associated with abnormal tissue level perfusion: a TACTICS-TIMI 18 substudy. Treat Angina with Aggrastat and Determine Cost of Therapy with an Invasive or Conservative Strategy-Thrombolysis in Myocardial Infarction. Circulation 2002;106:202-7.

21. Fuchs S, Stabile E, Mintz GS, et al. Intravascular ultrasound findings in patients with acute coronary syndromes with and without elevated troponin I level. Am J Cardiol 2002;89:1111-3.

22. Landesberg G, Shatz V, Akopnik I, et al. Association of cardiac troponin, CK-MB, and postoperative myocardial ischemia with long-term survival after major 
vascular surgery. J Am Coll Cardiol 2003;42:1547-54.

23. Kim LJ, Martinez EA, Faraday N, et al. Cardiac troponin I predicts short-term mortality in vascular surgery patients. Circulation 2002;106:2366-71.

24. Le Manach Y, Perel A, Coriat P, et al. Early and delayed myocardial infarction after abdominal aortic surgery. Anesthesiology 2005;102: 885-91.

25. Mahla E, Baumann A, Rehak P, et al. N-terminal pro-brain natriuretic peptide identifies patients at high risk for adverse cardiac outcome after vascular surgery. Anesthesiology 2007;106:1088-95.

26. Eagle KA, Rihal CS, Mickel MC, et al. Cardiac risk of noncardiac surgery: influence of coronary disease and type of surgery in 3368 operations. CASS Investigators and University of Michigan Heart Care Program. Coronary Artery Surgery Study. Circulation 1997;96:1882-7.

27. McFalls EO, Ward HB, Moritz TE, et al. Coronary-artery revascularization before elective major vascular surgery. N Engl J Med 2004;351:2795-804.

28. Eagle KA, Guyton RA, Davidoff RD, et al. ACC/AHA guidelines for coronary artery bypass graft surgery: executive summary and recommendations: a report of the American College of Cardiology/ American Heart Association Task Force on Practice Guidelines (Committee to Revise the 1991 Guidelines for Coronary Artery Bypass Graft Surgery). Circulation 1999;100:1464-80.

29. Landesberg G, Mosseri M, Wolf YG, et al. Preoperative thallium scanning, selective coronary revascularization, and long-term survival after major vascular surgery. Circulation 2003;108:177-83.

30. Garofalo M, Nardi P, Borioni R, et al. The impact of coronary revascularization on long-term outcomes after surgical repair of abdominal aortic aneurysm. Ital Heart J 2005;6(Suppl):369-74.

31. Ward HB, Kelly RF, Thottapurathu L, et al. Coronary artery bypass grafting is superior to percutaneous coronary intervention in prevention of perioperative myocardial infarctions during subsequent vascular surgery. Ann Thorac Surg 2006;82:795-800.

32. Hassan SA, Hlatky MA, Boothroyd DB, et al. Outcomes of noncardiac surgery after coronary bypass surgery or coronary angioplasty in the Bypass Angioplasty Revascularization Investigation (BARI). Am J Med 2001;110:260-6.

33. Holmes Jr DR, Savage M, LaBlanche JM, et al. Results of Prevention of REStenosis with Tranilast and its Outcomes (PRESTO) trial. Circulation 2002;106:1243-50. 
34. Hodgson JM, Bottner RK, Klein LW, et al. Drug-eluting stent task force: final report and recommendations of the working committees on costeffectiveness/economics, access to care, and medicolegal issues. Catheter Cardiovasc Interv 2004;62:1-17.

35. Tsimikas S. Drug-eluting stents and late adverse clinical outcomes lessons learned, lessons awaited. J Am Coll Cardiol 2006;47:2112-5.

36. Kaluza GL, Joseph J, Lee JR, et al. Catastrophic outcomes of noncardiac surgery soon after coronary stenting. J Am Coll Cardiol 2000;35:1288-94.

37. Leibowitz D, Cohen M, Planer D, et al. Comparison of cardiovascular risk of noncardiac surgery following coronary angioplasty with versus without stenting. Am J Cardiol 2006;97:1188-91.

38. Wilson SH, Fasseas P, Orford JL, et al. Clinical outcome of patients undergoing non-cardiac surgery in the two months following coronary stenting. J Am Coll Cardiol 2003;42:234-40.

39. Grines CL, Bonow RO, Casey Jr DE, et al. Prevention of premature discontinuation of dual antiplatelet therapy in patients with coronary artery stents: a science advisory from the American Heart Association, American College of Cardiology, Society for Cardiovascular Angiography and Interventions, American College of Surgeons, and American Dental Association, with representation from the American College of Physicians. Circulation 2007;115:813-8.

40. Vicenzi MN, Meislitzer T, Heitzinger B, et al. Coronary artery stenting and noncardiac surgery - a prospective outcome study. Br J Anaesth 2006;96:686-93.

41. Feringa HH, Bax JJ, Boersma E, et al. High-dose beta-blockers and tight heart rate control reduce myocardial ischemia and troponin $\mathrm{T}$ release in vascular surgery patients. Circulation 2006; 114:I344-9.

42. Raby KE, Brull SJ, Timimi F, et al. The effect of heart rate control on myocardial ischemia among high-risk patients after vascular surgery. Anesth Analg 1999;88:477-82.

43. Hindler K, Shaw AD, Samuels J, et al. Improved postoperative outcomes associated with preoperative statin therapy. Anesthesiology 2006;105:1260-72.

44. Durazzo AE, Machado FS, Ikeoka DT, et al. Reduction in cardiovascular events after vascular surgery with atorvastatin: a randomized trial. J Vasc Surg 2004;39:967-75.

45. Wallace AW, Galindez D, Salahieh A, et al. Effect of clonidine on cardiovascular 
morbidity and mortality after noncardiac surgery. Anesthesiology 2004;101:28493.

46. Burger W, Chemnitius JM, Kneissl GD, et al. Low-dose aspirin for secondary cardiovascular prevention-cardiovascular risks after its perioperative withdrawal versus bleeding risks with its continuation - review and meta-analysis. J Intern Med 2005;257:399-414.

47. Mangano DT. Aspirin and mortality from coronary bypass surgery. N Engl J Med 2002;347:1309-17.

48. Bybee KA, Powell BD, Valeti U, et al. Preoperative aspirin therapy is associated with improved postoperative outcomes in patients undergoing coronary artery bypass grafting. Circulation 2005;112: I286-92.

49. Robless P, Mikhailidis DP, Stansby G. Systematic review of antiplatelet therapy for the prevention of myocardial infarction, stroke or vascular death in patients with peripheral vascular disease. Br J Surg 2001;88:787-800.

50. Prevention of pulmonary embolism and deep vein thrombosis with low dose aspirin: Pulmonary Embolism Prevention (PEP) trial. Lancet 2000;355:1295-302.

51. Yang H, Raymer K, Butler R, et al. The effects of perioperative beta-blockade: results of the Metoprolol after Vascular Surgery (MaVS) study, a randomized controlled trial. Am Heart J 2006;152:983-90.

52. Juul AB, Wetterslev J, Gluud C, et al. Effect of perioperative beta blockade in patients with diabetes undergoing major non-cardiac surgery: randomised placebo controlled, blinded multicentre trial. BMJ 2006;332:1482.

53. Brady AR, Gibbs JS, Greenhalgh RM, et al. Perioperative beta-blockade (POBBLE) for patients undergoing infrarenal vascular surgery: results of a randomized double-blind controlled trial. J Vasc Surg 2005;41:602-9.

54. McCullough PA. Failure of beta-blockers in the reduction of perioperative events: where did we go wrong? Am Heart J 2006;152:815-8.

55. Devereaux PJ, Yang H, Guyatt GH, et al. Rationale, design, and organization of the PeriOperative ISchemic Evaluation (POISE) trial: a randomized controlled trial of metoprolol versus placebo in patients undergoing noncardiac surgery. Am Heart J 2006;152:223-30.

56. Schouten O, Poldermans D, Visser L, et al. Fluvastatin and bisoprolol for the reduction of perioperative cardiac mortality and morbidity in high-risk patients undergoing non-cardiac surgery: rationale and design of the DECREASE-IV study. Am Heart J 2004;148:1047-52. 
57. Ellison KE, Gandhi G. Optimising the use of beta-adrenoceptor antagonists in coronary artery disease. Drugs 2005;65:787-97 44.

58. Fox KA, Steg PG, Eagle KA, et al. Decline in rates of death and heart failure in acute coronary syndromes, 1999-2006. JAMA 2007;297:1892-900.

59. Lindenauer PK, Pekow P, Wang K, et al. Perioperative beta-blocker therapy and mortality after major noncardiac surgery. N Engl J Med 2005;353:349-61.

60. Pasternak RC, Smith Jr SC, Bairey-Merz CN, et al. ACC/AHA/NHLBI clinical advisory on the use and safety of statins. Circulation 2002;106:1024-8. 